\title{
A Study on the Risk Regulation of Financial Investment Market Based on Quantitative
}

\author{
Xinfeng $\mathrm{Li}^{1}$, Hao Deng ${ }^{* 2}$ \\ ${ }^{1}$ College of Liberal Arts, University of Minnesota, Twin Cities, Minneapolis, The United States \\ ${ }^{2}$ School of Mathematics, University of Edinburgh, Edinburgh, The United Kingdom \\ *Email: Li000027@umn.edu; 564398527@qq.com
}

\begin{abstract}
To better supervise the risk of financial investment market, the Conditional Value At Risk (CVaR) investment portfolio optimization model of under single period investment is discussed and its expansion form is explained. The empirical simulation of Mean - CVaR model is carried out with historical simulation method, and the influence of confidence level and transaction cost on the effective frontier of the optimization model is mainly studied. The results show that the effective frontier of the Mean - CVaR model will move to the right as the confidence level increases and will also move to the lower right as the transaction cost increases. In addition, the empirical simulation part also confirms the rationality of the mean model and summarizes the relationship between -CVaR and - Value At Risk (VaR) and the characteristics of their respective effective frontier curves. It can be concluded that the Mean - CVaR model has a good effect on the risk supervision of financial investment market.
\end{abstract}

Keywords: Quantitative model; financial investment market; risk supervision; CVaR model

\section{Introduction}

Investment in tradable financial assets is a double-edged sword. From a management perspective, many companies hold tradable financial assets, which indeed increases the liquidity of assets or the profitability of spare assets; however, from a risk perspective, many companies hold tradable financial assets, which indeed increases the risk of value volatility and asset load. With the rapid development of global capital markets, financial instruments and derivative financial instruments have become increasingly rich and complex. How to define and select tradable financial assets and combine them is the problem that the company has to consider in investment management. And with the increase in the price fluctuation of tradable financial assets, how to prevent and control the potential risks of the investment of tradable financial assets and how to ensure the matching of the investment portfolio with its own risk tolerance and control ability also become a problem that can't be ignored by the company in risk management. Based on the practical needs, the investment strategy and financial risk prevention of tradable financial assets are studied. It aims to improve the company's investment management ability and risk prevention and control ability by strengthening the attention and management of financial indicators related to the investment of tradable financial assets.

\section{Literature Review}

In 2015, Theobald (2015) established a mathematical model - mean variance model, which connected the opposite characteristics of the profitability and risk of assets, he discussed the choice of optimal investment portfolio under uncertain conditions, leading the financial investment into the era of quantitative analysis. The mean-variance model provided a research framework for portfolio optimization theory. Once the model was proposed, scholars had tried to apply it to practice [1]. However, due to the underdeveloped computer technology at the time, the solution of the mean-variance model was extremely difficult. Therefore, to improve the efficiency of parameter estimation for this vast system engineering, Detzer (2015) created the Single Index Model. At the same time, to facilitate the calculation of large-scale optimization problems [2], Davis (2015) used the average absolute deviation to measure the risk of portfolio and built the Mean Absolute Deviation Model that can be transformed into 
linear programming problems [3]. In addition, there was a defect in using variance to measure risk, that is, when the distribution of returns was asymmetric, different portfolios had different skewness for the same mean and variance; this means that the variance is not a complete indicator of risk. Therefore, Haifan (2015) added skewness target into the traditional mean variance model and constructed the Multi View System (MVS) model. But it was not the convex programming, and it was very hard to solve [4].

As a risk measurement index, the variance not only reflected the loss situation where the rate of return was lower than the expected value, but also included the earnings situation where the rate of return was higher than the expected value, which obviously didn't match people's psychological expectation of the risk. For this reason, scholars put forward the following risk measurement indicators and tried to establish the relevant new portfolio optimization model. For example, Luo (2015) proposed to measure risk with the downside semi-variance and discussed the mean variance model [5]; Kopp (2017) measured risk with Linear Probability Model (LPM) and established the mean - lower partial moment model [6]. However, the above risk measure index didn't intuitively reflect the scale of the risks faced by investors. Therefore, in today's society where financial risks are particularly complicated, both VaR and CVaR can reasonably estimate the loss value faced by investors at a certain level of confidence, and these two risk measure indexs were naturally applied to portfolio optimization. Grosse (2017) established an optimization model by measuring the risk of the portfolio [7]. Subsequently, Lin (2018) pointed out that when the return on assets doesn't obey the elliptic distribution, VaR can't be expressed as a smooth convex function of the asset position, and the mean-VaR model has multiple extremum problems. In addition, relevant scholars introduced the consistency risk measurement standard, and proved that the VaR index didn't meet the sub-additivity, which indicated that the mean-VaR model had technical difficulty in calculation. As a result, relevant researchers built a CVaR portfolio optimization model that is easy to implement and extend [8].

\section{Methodology}

\subsection{Historical Simulation Method}

Historical simulation method is a method to describe the future changes of the market factors by selecting the past data that can represent the fluctuations of the market factors within a period of time, and then use the relationship between asset value and risk factors to fit the return distribution of the assets. This method is simple to operate and easy to implement. Therefore, many financial institutions use it to fit the probability distribution of market factors. Suppose portfolio P contains $\mathrm{n}$ securities and wi $(\mathrm{i}=1, \ldots, \mathrm{n})$ represents the investment weight of various assets. The steps to estimate the CVaR value of a portfolio with historical simulation method are as follows:

Step 1: determine $\mathrm{k}$ risk factors influencing the change of portfolio value, and estimate the daily variation of each factor;

Step 2: predict $m$ possible future loss scenarios of the portfolio based on the relationship between the fitted portfolio value and the market factor price;

Step 3: estimate the daily VaR value and daily CVaR value of the portfolio.

However, the historical simulation method has many shortcomings. For this reason, relevant scholars created the BRW method. This method combines the historical simulation method and the exponential smoothing technology to calculate the VaR value of the portfolio. Here, the main purpose of introducing exponential smoothing technology is to estimate the correlation and conditional volatility of return sequences. The core idea of the implementation of exponential smoothing technology is: it thinks that the time series data of different time periods has different effects on the determination of profit and loss distribution. Therefore, different weights should be selected for these data; the closer the data is to the current period, the greater the weight, and vice versa.

\subsection{Monte Carlo Simulation Method}

The Monte Carlo simulation method needs to set the distribution function in advance, and then simulates the random trend of the financial variables by repeatedly generating and extracting random 
numbers. In recent years, this method has been widely used with the rapid development of computer technology. The following is a description of the implementation steps of the Monte Carlo simulation method for a single random rate of return variable.

Step 1: select the random model, so that the stock price data generated by the model is basically consistent with the actual performance of the market.

Step 2: generate a series of random numbers $\varepsilon$ that follow the standard normal distribution $N(0,1)$, and then repeatedly extract random values and plug them into the model set in step 1 to obtain $\mathrm{m}+1$ stock price scenarios.

Step 3: repeat the simulated operation in step 2 for many times, and then calculate the price sequence of the asset portfolio at the target time point, and finally obtain the VaR value of this time point.

The Monte Carlo simulation method also has many defects. For this reason, scholars have put forward the Quasi-Monte Carlo simulation method that replaces pseudo-random number sequence with quasi-random sequence of numbers, the scenario simulation method that uses principal component analysis to reduce the dimension of market factor, and the Markov Chain Monte Carlo simulation method (ie MCMC method) that combines the Markov process with the Monte Carlo simulation method to improve the computational efficiency and computational accuracy of the traditional Monte Carlo simulation method.

\section{Empirical Analysis}

\subsection{Selection of Sample Data and Analysis of Statistical Characteristics}

Eight stocks (ie $n=8$ ) are randomly selected from the two stock markets of Shanghai and Shenzhen in China as the research objects. The eight stocks are in different industries, including Ping An Bank, Yantian Port, Yunnan Baiyao, Wuliangye, Minmetals Development, China Television Media, Youngor, Shandong Nanshan Aluminium. At the same time, historical simulation method will be adopted to generate the rate of return scenarios of various financial assets. The time span of the selected samples is from June 1, 2015 to June 15, 2017, for a total of 495 trading days, that is, the number of selected scenarios is $495, \mathrm{~m}=495$.

The stock data used in the empirical model are data of daily rate of return. Its calculation method is the logarithmic difference between the closing price and the opening price of each trading day, that is, the jth yield scenario of the ith stock is:

$$
\begin{gathered}
r_{i j}=\ln \frac{P_{i j}^{2}}{2} ; i=1,2 \ldots, n, j=1,2, \ldots, m \\
P_{i j}^{1}
\end{gathered}
$$

Here, $\mathrm{p}_{\mathrm{ij}}{ }^{2}$ represents the closing price of stock $\mathrm{i}$ on trading day $\mathrm{j}, \mathrm{p}_{\mathrm{ij}}{ }^{1}$ represents the opening price of stock $\mathrm{i}$ on trading day $\mathrm{j}$. In addition, if the stock's data on a trading day is missing, its data of rate of return for that day is replaced by its average daily rate of return. All raw data used are derived from the CSMAR database.

Table 1. Descriptive statistical results of data of daily rate of return for 8 sample stocks

\begin{tabular}{ccccccc}
\hline Stock name & Mean & Standard deviation & Skewness & Kurtosis & JB statistics & P value \\
\hline Ping An Bank & 0.00022 & 0.0151 & 0.2966 & 4.7654 & 71.5411 & 0 \\
Yantian Port & 0.00059 & 0.0162 & -0.1158 & 7.2345 & 370.9264 & 0 \\
Yunnan Baiyao & 0.00124 & 0.0182 & 0.0735 & 4.7782 & 65.6581 & 0 \\
Wuliangye & 0.00095 & 0.0181 & -0.0226 & 3.3155 & $2.0955^{*}$ & $0.35^{*}$ \\
Minmetals Development & 0.00291 & 0.0306 & 0.33713 & 4.4194 & 52.9313 & 0 \\
China Television Media & 0.00173 & 0.0281 & 0.1429 & 4.9562 & 80.6159 & 0 \\
Youngor & 0.00027 & 0.0180 & -0.0742 & 3.7768 & 12.8980 & 0.002 \\
Shandong Nanshan Aluminium & 0.00018 & 0.0198 & 0.4277 & 6.5434 & 274.0435 & 0 \\
\hline
\end{tabular}

According to formula (1), the LN function in Excel 2013 can be called to obtain the daily return series of a single stock. Then, the descriptive statistical characteristics of the data of daily rate of return of 
these 8 stocks are obtained with Eviews 5.0 analysis, as shown in table 1.0 .05 is taken as the significance level of the Jarque-Bera test. At this moment, the critical value of the test is 5.9495, which means that the daily rate of return sequences of all 7 stocks except Wuliangye are significantly not subject to normal distribution.

\subsection{Simulation Analysis of Mean -CVaR Model}

The scenarios of return rate of 8 securities obtained by historical simulation method are used to simulate and analyze the CVaR portfolio optimization model. The confidence level in the model is $0.85,0.90,0.95$ and 0.99 . To more clearly use mathematical software to solve the model, all the variables involved in the model are analyzed in table 2 below.

Table 2. Analysis of the properties of the variables in the CvaR portfolio optimization model

\begin{tabular}{|c|c|c|c|c|}
\hline Symbol & Nature & Element or value range & Dimension & $\begin{array}{l}\text { Input/output } \\
\text { attribute }\end{array}$ \\
\hline $\mathrm{w}$ & The weight vector of the portfolio & $\mathrm{w}_{\mathrm{i}}$ & $\mathrm{n} \times 1$ & Output variable \\
\hline $\mathrm{r}$ & The vector of random rate of return & $\mathrm{r}_{\mathrm{i}}$ & $\mathrm{n} \times 1$ & Input parameter \\
\hline$r_{j}$ & The scenario of rate of return on assets & $\mathrm{r}_{\mathrm{ij}}$ & $\mathrm{n} \times \mathrm{m}$ & Input parameter \\
\hline $\mathrm{z}$ & Auxiliary variable & $z_{i}$ & $\mathrm{~m} \times 1$ & Output variable \\
\hline$\eta$ & VaR value in a portfolio & This value & $1 \times 1$ & Output variable \\
\hline $\mathrm{p}$ & $\begin{array}{l}\text { The probability of the rate of return } \\
\text { scenario }\end{array}$ & $\begin{array}{l}\mathrm{P}^{\mathrm{j}} \text { (set as equally likely, } \\
\qquad 1 / \mathrm{m})\end{array}$ & $\mathrm{m} \times 1$ & Calculate in advance \\
\hline$r_{p}$ & The expected rate of return on the portfolio & Interval $[0.006,0.0029]$ & $1 \times 1$ & Select in advance \\
\hline$\alpha$ & Confidence level & $0.85,0.90,0.95,0.99$ & $1 \times 1$ & Select in advance \\
\hline $\mathrm{n}$ & The number of assets in a portfolio & Set to 8 & $1 \times 1$ & Determine in advance \\
\hline $\mathrm{m}$ & Number of scenarios for the rate of return & Set to 495 & $1 \times 1$ & Determine in advance \\
\hline
\end{tabular}

In the following, the optimization model is solved with the instruction for solving linear programming in Matlab 6.5, and the obtained correspondence of mean-CVaR and the optimal investment weight result are shown in Table 3 . In addition, table 4 shows the relationship between the average value of unit risk return and confidence coefficient.

Table 3. The optimal investment weight of the CvaR portfolio optimization model

\begin{tabular}{|c|c|c|c|c|c|c|c|c|}
\hline \multirow{9}{*}{$\begin{array}{c}\text { Confidence } \\
\text { level } \\
\alpha=0.85\end{array}$} & $\mathrm{w}_{1}$ & 0.29 & 0.19 & 0.12 & 0 & 0 & 0 & 0 \\
\hline & $\mathrm{w}_{2}$ & 0.16 & 0.15 & 0.11 & 0.10 & 0 & 0 & 0 \\
\hline & $\mathrm{w}_{3}$ & 0.11 & 0.24 & 0.29 & 0.31 & 0.23 & 0 & 0 \\
\hline & $\mathrm{w}_{4}$ & 0.05 & 0.04 & 0.02 & 0.02 & 0 & 0 & 0 \\
\hline & $\mathrm{w}_{5}$ & 0.04 & 0.12 & 0.23 & 0.34 & 0.50 & 0.74 & 1 \\
\hline & $\mathrm{w}_{6}$ & 0.04 & 0.07 & 0.15 & 0.21 & 0.27 & 0.26 & 0 \\
\hline & $\mathrm{w}_{7}$ & 0.12 & 0.07 & 0.01 & 0 & 0 & 0 & 0 \\
\hline & $\mathrm{w}_{8}$ & 0.18 & 0.11 & 0.08 & 0.03 & 0 & 0 & 0 \\
\hline & Number of assets & 8 & 8 & 8 & 6 & 3 & 3 & 1 \\
\hline \multirow{9}{*}{$\begin{array}{c}\text { Confidence } \\
\text { level } \\
\alpha=0.90\end{array}$} & $\mathrm{w}_{1}$ & 0.28 & 0.22 & 0.14 & 0.01 & 0 & 0 & 0 \\
\hline & $\mathrm{w}_{2}$ & 0.18 & 0.13 & 0.10 & 0.11 & 0 & 0 & 0 \\
\hline & $\mathrm{w}_{3}$ & 0.13 & 0.21 & 0.20 & 0.29 & 0.23 & 0 & 0 \\
\hline & $\mathrm{w}_{4}$ & 0.05 & 0.04 & 0.08 & 0.06 & 0 & 0 & 0 \\
\hline & $\mathrm{w}_{5}$ & 0.04 & 0.13 & 0.25 & 0.34 & 0.50 & 0.74 & 1 \\
\hline & $\mathrm{w}_{6}$ & 0.03 & 0.09 & 0.15 & 0.19 & 0.27 & 0.26 & 0 \\
\hline & $\mathrm{w}_{7}$ & 0.13 & 0.08 & 0 & 0 & 0 & 0 & 0 \\
\hline & $\mathrm{w}_{8}$ & 0.16 & 0.10 & 0.09 & 0 & 0 & 0 & 0 \\
\hline & Number of assets & 8 & 8 & 7 & 6 & 3 & 2 & 1 \\
\hline
\end{tabular}




\begin{tabular}{|c|c|c|c|c|c|c|c|c|}
\hline \multirow{9}{*}{$\begin{array}{c}\text { Confidence } \\
\text { level } \\
\alpha=0.85\end{array}$} & $\mathrm{w}_{1}$ & 0.31 & 0.29 & 0.19 & 0.07 & 0 & 0 & 0 \\
\hline & $\mathrm{w}_{2}$ & 0.19 & 0.19 & 0.17 & 0.05 & 0 & 0 & 0 \\
\hline & $\mathrm{w}_{3}$ & 0.11 & 0.19 & 0.26 & 0.30 & 0.23 & 0 & 0 \\
\hline & $\mathrm{w}_{4}$ & 0.08 & 0.02 & 0 & 0 & 0 & 0 & 0 \\
\hline & $\mathrm{w}_{5}$ & 0.04 & 0.14 & 0.23 & 0.33 & 0.49 & 0.74 & 1 \\
\hline & $\mathrm{w}_{6}$ & 0.03 & 0.08 & 0.15 & 0.24 & 0.28 & 0.26 & 0 \\
\hline & $\mathrm{w}_{7}$ & 0.15 & 0.06 & 0 & 0 & 0 & 0 & 0 \\
\hline & $\mathrm{w}_{8}$ & 0.09 & 0.03 & 0 & 0 & 0 & 0 & 0 \\
\hline & Number of assets & 8 & 8 & 5 & 5 & 3 & 2 & 1 \\
\hline \multirow{9}{*}{$\begin{array}{c}\text { Confidence } \\
\text { level } \\
\alpha=0.85\end{array}$} & $\mathrm{w}_{1}$ & 0.36 & 0.32 & 0.19 & 0 & 0 & 0 & 0 \\
\hline & $\mathrm{w}_{2}$ & 0.25 & 0.15 & 0.21 & 0.21 & 0 & 0 & 0 \\
\hline & $\mathrm{w}_{3}$ & 0.15 & 0.21 & 0.24 & 0.30 & 0.19 & 0 & 0 \\
\hline & $\mathrm{w}_{4}$ & 0 & 0 & 0 & 0 & 0 & 0 & 0 \\
\hline & $\mathrm{W}_{5}$ & 0.03 & 0.12 & 0.26 & 0.39 & 0.48 & 0.74 & 1 \\
\hline & $\mathrm{w}_{6}$ & 0.03 & 0.13 & 0.11 & 0.10 & 0.32 & 0.26 & 0 \\
\hline & $\mathrm{w}_{7}$ & 0.18 & 0.07 & 0 & 0 & 0 & 0 & 0 \\
\hline & $\mathrm{w}_{8}$ & 0 & 0 & 0 & 0 & 0 & 0 & 0 \\
\hline & Number of assets & 6 & 6 & 5 & 4 & 3 & 2 & 1 \\
\hline
\end{tabular}

Table 4. Correspondence between the average value of unit risk return and confidence coefficient

\begin{tabular}{|c|c|c|c|c|c|c|c|}
\hline Confidence coefficient & 0.99 & 0.95 & 0.90 & 0.85 & 0.80 & 0.70 & 0.60 \\
\hline Average of $\mathrm{r}_{\mathrm{p}} / \mathrm{CVaR}$ & 0.039 & 0.057 & 0.070 & 0.081 & 0.091 & 0.114 & 0.140 \\
\hline Average of $\mathrm{r}_{\mathrm{p}} / \mathrm{VaR}$ & 0.048 & 0.076 & 0.105 & 0.135 & 0.172 & 0.308 & 0.721 \\
\hline
\end{tabular}

Combined with table 3 and table 4, the following comparative analysis results can be obtained:

Firstly, when the expected return rate of the portfolio is fixed, both the value of CVaR and VaR increase with the increase of confidence level, and both $r_{p} / C V a R$ and $r_{p} / \mathrm{VaR}$ decrease with the increase of confidence level, appearing at the effective frontier of the mean-CVaR and the mean-VaR, as shown in Figure 1 and Figure 2. That is, both efficient frontiers are shifted to the right as the confidence level increases, meaning that the degree of risk aversion of investors increases as the confidence level increases.

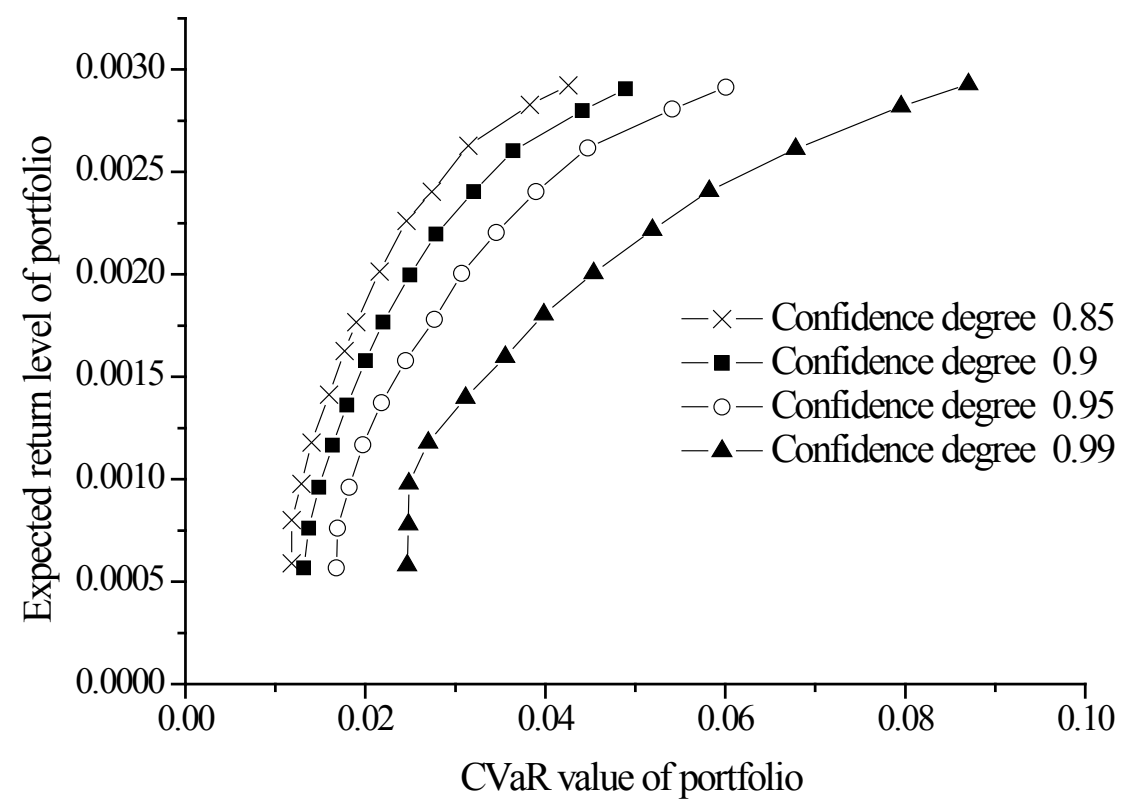

Figure 1. The effective frontier of the mean -CVaR model at different confidence levels 
Secondly, when the confidence level is fixed, the CVaR value of the portfolio increases with the increase of expected return rate. That is, if the risk-averse investor wants to reduce the probability of tail risk by increasing the confidence level, he will face the fact that the expected rate of return also decreases, which means that the returns and risks depicted by the CVaR optimization model also conform to the characteristics of positive correlation. This is reflected in the effective frontier of the optimization model, that is, its effective frontier curve is smooth and monotonic nondecreasing, as shown in figure 1. On the other hand, when the confidence level is fixed, the VaR value of the portfolio doesn't necessarily increase with the increase of expected return rate. This is shown in the effective frontier of the mean-VaR, that is, this effective frontier curve is not monotonic nondecreasing in some areas, as shown in Figure 2.

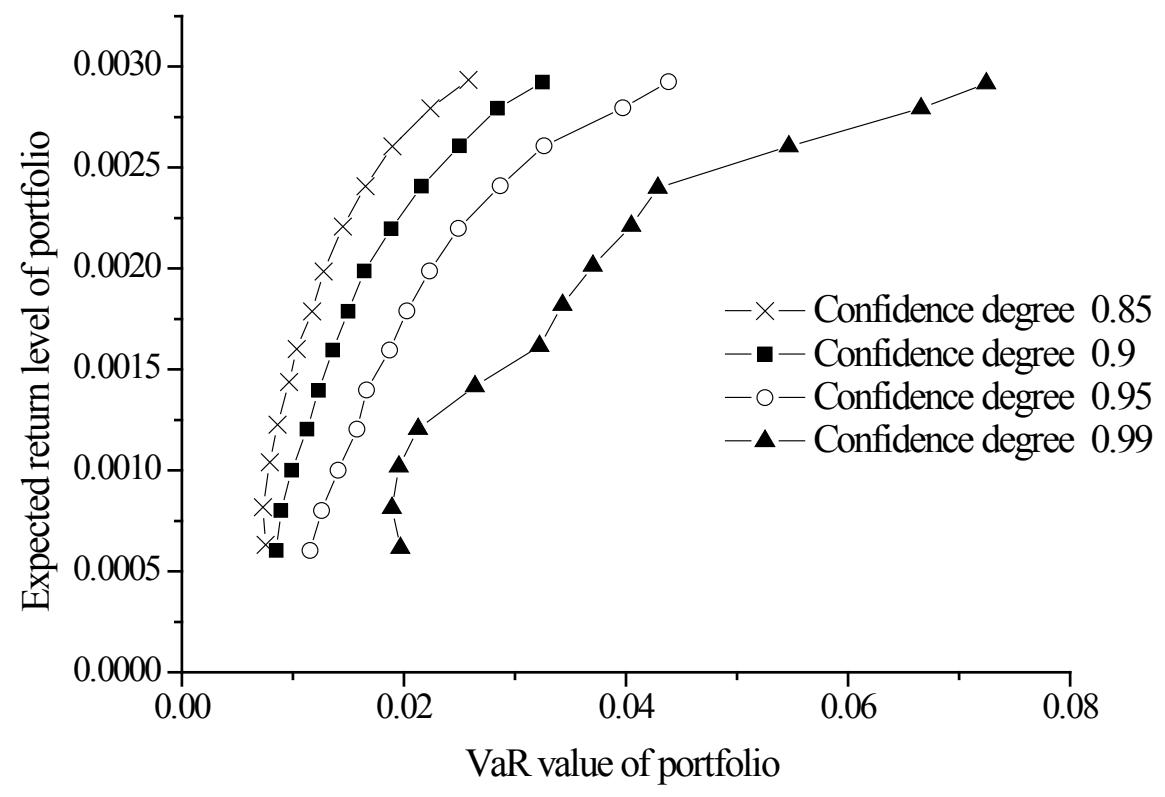

Figure 2. Effective frontier of the mean -VaR model at different confidence levels

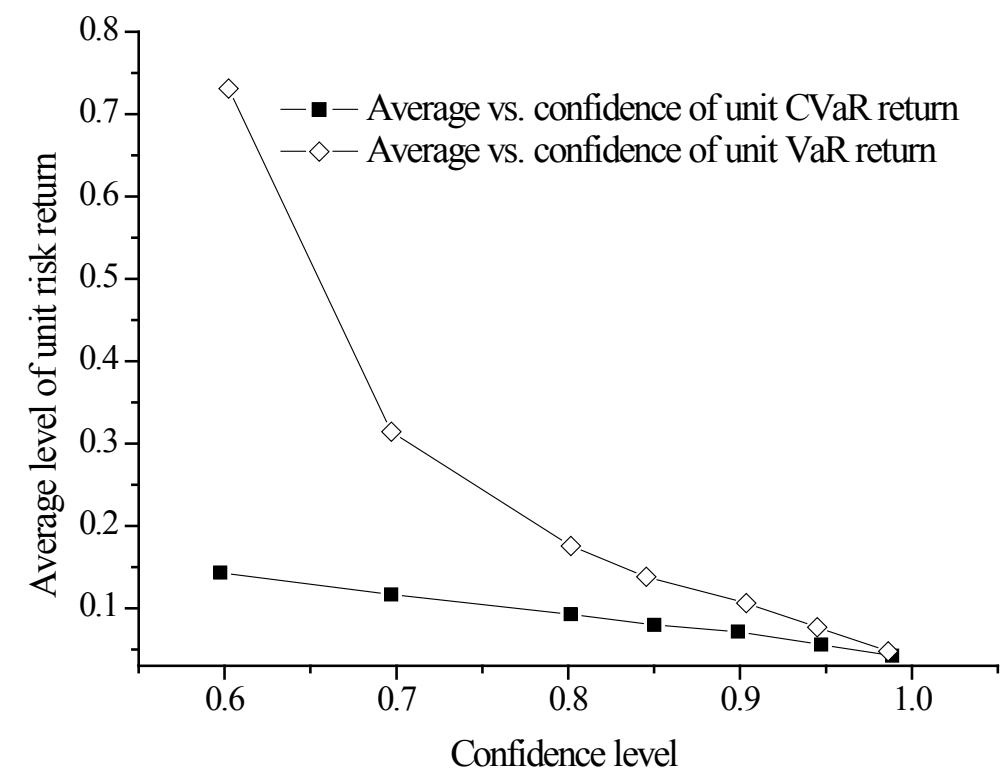

Figure 3. Correspondence diagram between the average of return per unit risk and confidence level 
Thirdly, the average of the unit CVaR returns and the average of the unit VaR returns decrease as the confidence level increases, as shown in Figure 3. This means that when the expected rate of return of the portfolio is fixed, both the CVaR value and $\mathrm{VaR}$ value increase with the increase of confidence level. This conclusion just confirms the first point.

Fourthly, when the expected rate of return of the portfolio is fixed, the value of CVaR is always higher than the value of $\mathrm{VaR}$, which is shown in figure 4, that is, the CVaR curve is always on the right side of the VaR curve. This is consistent with the definition of CVaR, that is, the loss at CVaR exceeds the expected loss at VaR.

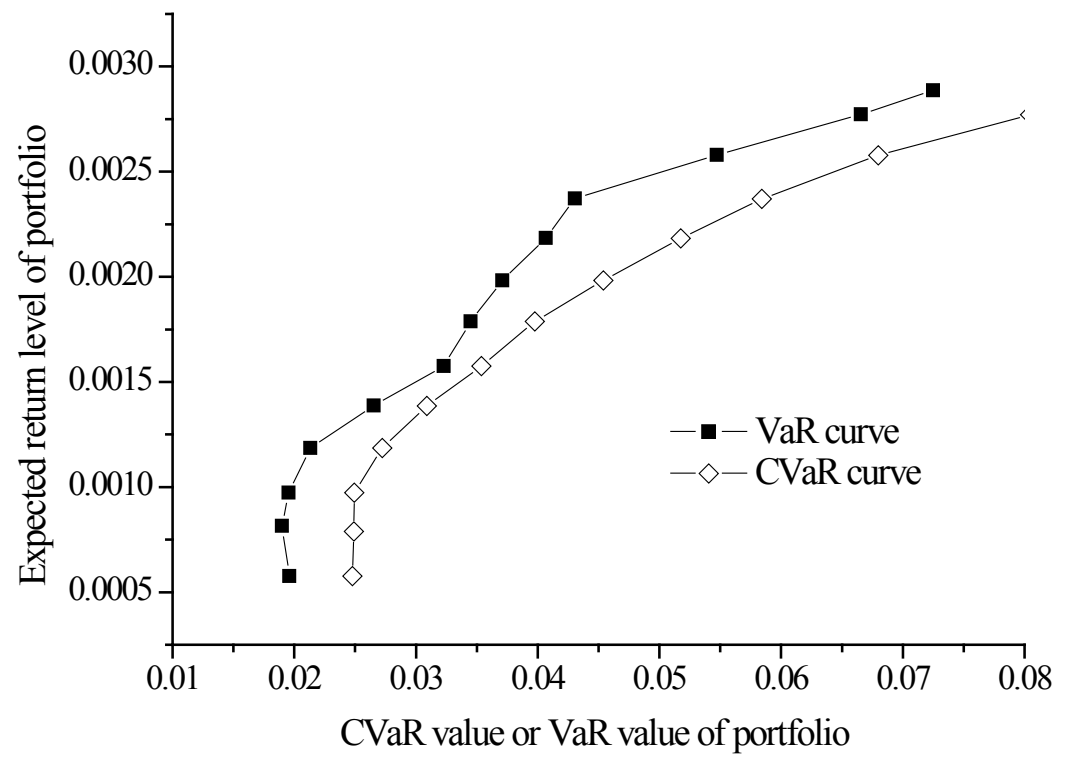

Figure 4. The CVaR curve and VaR curve at the 0.99 confidence level

Fifthly, with the gradual increase of the expected return rate of the portfolio and the gradual increase of the CVaR value of the portfolio, the number of assets contained in the portfolio is gradually reduced, that is, the investment is more concentrated, as shown in figures 5 and 6 . This is consistent with the principle of investment diversification and risk diversification.

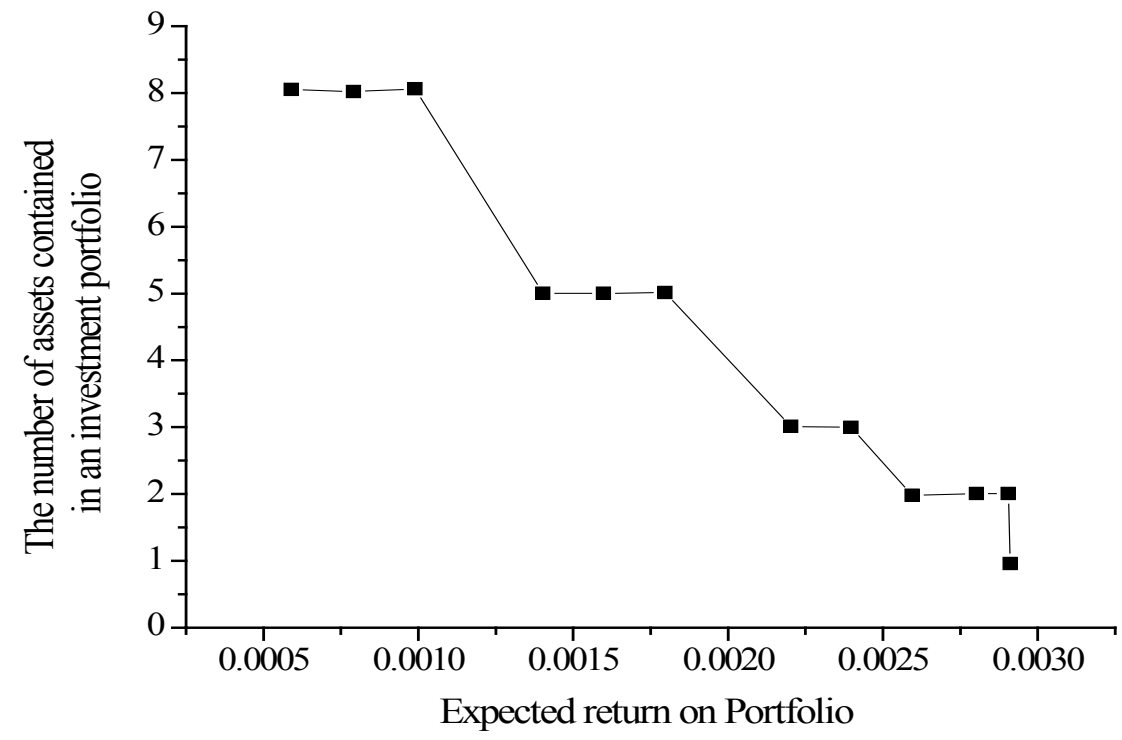

Figure 5. Correspondence diagram between the expected rate of return and the number of assets in a portfolio (the confidence level is 0.95 ) 


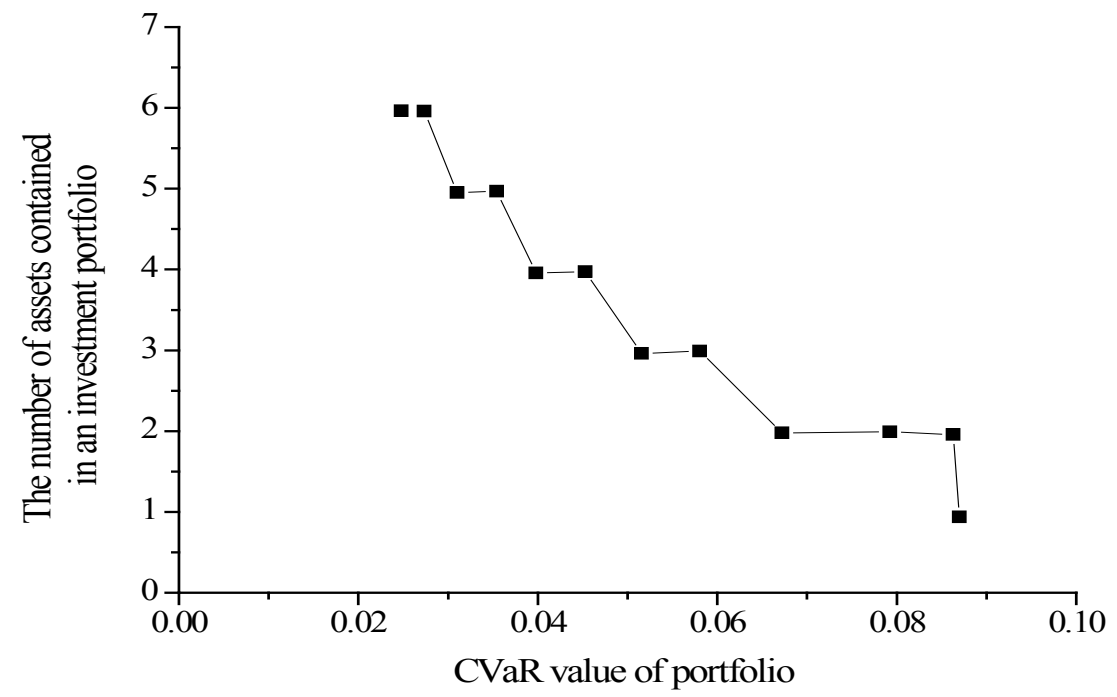

Figure 6. Correspondence diagram between the CVaR value and the number of assets in a portfolio (the confidence level is 0.99)

\subsection{Analysis of the Impact of Transaction Costs on the Efficient Frontier of Portfolio Optimization Model}

When trading A shares in China's Shanghai Stock Exchange and Shenzhen Stock Exchange, investors often need to pay commission fees, commissions, stamp tax and transfer fees, etc. Therefore, the CVaR portfolio optimization model with transaction cost has certain guiding significance for investors to construct the optimal portfolio in real trading.

The data of rate of return used in the empirical simulation is daily data, and in general, the daily expected return level is relatively low. Therefore, to make the expected return of optimal portfolio not to be negative, the rate of transaction costs is specifically reduced, the effect of transaction cost on the effective frontier of CVaR portfolio optimization model is studied only by considering four situations where unit transaction cost $\mathrm{k}$ is $0.0003,0.0005,0.001$ and 0.0015 , and it is compared with the case when $\mathrm{k}=0$, that is, when there is no transaction cost. At the same time, since the main objective of this section is to explore how transaction costs will affect the effective frontier of the mean-CVaR model and the investor's optimal investment strategy, the conclusions obtained are still valid after using the above method to deal with transaction costs. In addition, the confidence level is set to 0.85 , that is, $\alpha=0.85$, and the initial holding ratio of each financial asset is set to 0 . The instruction for solving the linear programming is called to solve the optimization model, and the following results can be obtained.

Firstly, when the confidence level is fixed, the CVaR value of the portfolio increases with the increase of the unit transaction cost for the same expected return level. This means that the overall risk return level of the portfolio declines with the increase of transaction costs, which is reflected in the effective frontier of the mean-CVaR model with transaction costs, that is, the effective frontier moves to the right with the increase of transaction costs, as shown in figure 7.

Secondly, the upper and lower limits of the expected rate of return of the portfolio are smaller with the rise of transaction costs, and the range of the expected rate of return of the portfolio available for investors is smaller with the increase of transaction costs. This means that the upper and lower limits of the effective frontier of the CVaR portfolio optimization model with transaction costs decrease with the increase of unit transaction costs, which is reflected in the effective frontier of the average model with transaction costs, that is, the effective frontier moves to the lower right with the increase of transaction costs, as shown in figure 7. 


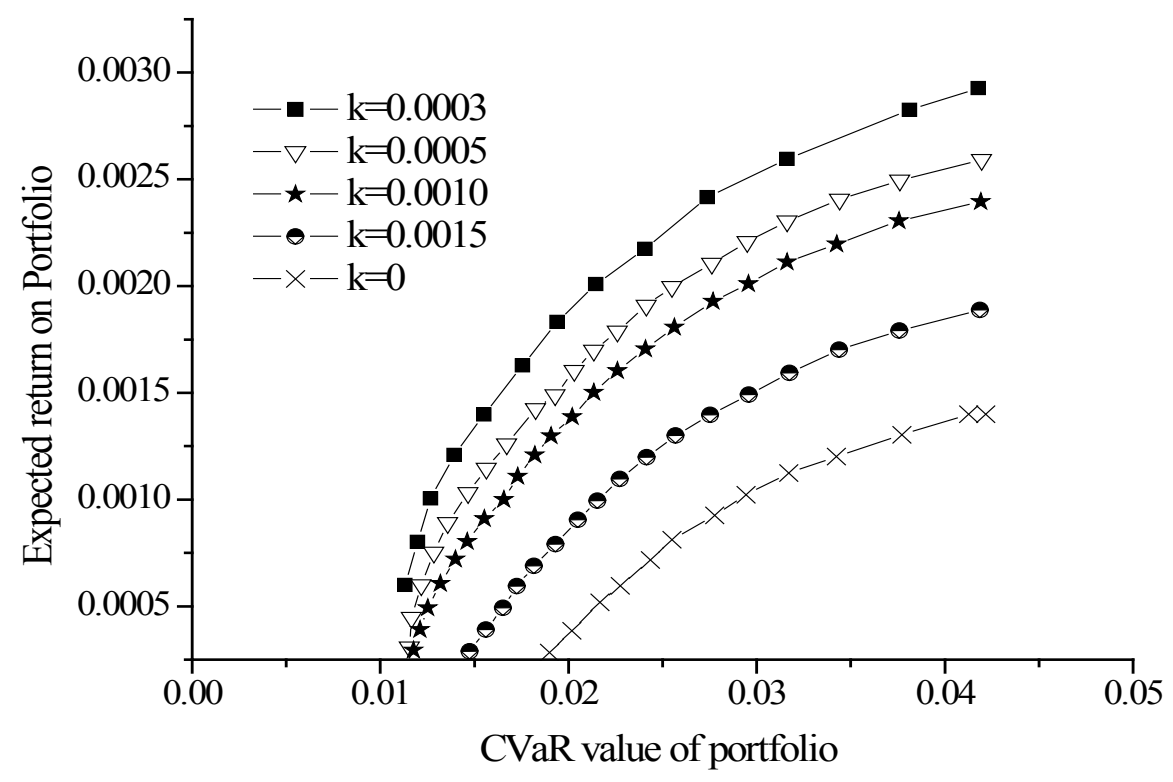

Figure 7. The efficient frontier of the average -CVaR model with transaction costs (the confidence level is 0.95 )

Thirdly, for any feasible expected return level of the portfolio, the type of asset composition and investment proportion of the portfolio will change significantly with the increase of transaction costs, which means that the optimal investment strategy of investors will be affected by the existence of transaction costs. This conclusion is a natural extension of the above two points.

\section{Conclusion}

The optimization model of CVaR portfolio under single-period investment is discussed and its expansion form is explained. The solution of the optimization model aiming at controlling the CVaR value of portfolio can be attributed to convex programming problem, while the solution of the mean-CVaR model can be transformed into the linear programming problem under the limited scenario of random rate of return variable, which not only avoids the problem of multiple extreme values, but also facilitates the solution and expansion of the model. This not only avoids the multiple extremum, but also facilitates the solution and expansion of the model.

In addition, the historical simulation method is used to simulate the mean-CVaR model, and the influence of confidence level and transaction cost on the effective frontier of the optimization model is studied. The results show that the effective frontier of the mean-CVaR model will move to the right as the confidence level increases and will also move to the lower right as the transaction cost increases. In addition, the rationality of the mean model is also confirmed, and the relationship between -CVaR and $\mathrm{VaR}$ and the characteristics of the respective effective frontier curves are summarized.

\section{References}

1. Theobald T. Agent-based risk management - A regulatory approach to financial markets. Imk Working Paper, 2015, 42(5), 780-820.

2. Detzer D K. Financial Market Regulation in Germany - Capital Requirements of Financial Institutions. Social Science Electronic Publishing, 2015, 68(272), 57-87.

3. Davis K. Competition and Financial Regulation. Australian Economic Review, 2015, 48(2), 180-185.

4. Haifan X. Introduction of Integrated Risk Management Paradigm of Financial Regulation. Finance \& Economics, 2015, 5, 327-328. 
5. Luo C, Chi X, Cong Y, et al. Measuring financial market risk contagion using dynamic MRS-Copula models: The case of Chinese and other international stock markets. Economic Modelling, 2015, 51, 657-671.

6. Kopp E, Kaffenberger L, Jenkinson N. Cyber Risk, Market Failures, and Financial Stability. Imf Working Papers, 2017, 17(185), 1.

7. Grosse, Robert. The global financial crisis - Market misconduct and regulation from a behavioral view. Research in International Business \& Finance, 2017, 41, 387-398.

8. Lin E, Sun E W, Yu M T. Systemic Risk, Financial Markets, and Performance of Financial Institutions. Annals of Operations Research, 2018, 262(2), 579-603. 